\title{
Title: Analysis of physical demands during youth soccer match-play: Considerations of sampling method and epoch length
}

Authors: Greg Doncaster ${ }^{1}$, Richard Page ${ }^{1}$, Paul White ${ }^{2}$, Robert Svenson ${ }^{2}$, Craig Twist ${ }^{3}$.

Institutional Affiliations: ${ }^{1}$ Edge Hill University (UK),

${ }^{2}$ Stoke City FC Academy, Stoke-on-Trent, UK.

${ }^{3}$ University of Chester, Chester, UK. Email: c.twist@ chester.ac.uk

Corresponding Author: Dr. Greg Doncaster

Department of Physical Activity \& Sport,

Faculty of Arts \& Sciences,

Edge Hill University, Ormskirk,

L39 4QP,

01695584151 ,

doncasg@edgehill.ac.uk

No funding or IRB approval was required for this research study.

\section{Acknowledgements}

The authors would like to thank the players, coaches and staff in their support of this study. No source of funding was obtained for this study and the authors have no conflicts of interest to declare.

Keywords: Small-Sided-Games; GPS; Monitoring Performance; Conditioning 


\title{
Title: Analysis of physical demands during youth soccer match-
}

\section{play: Considerations of sampling method and epoch length}

\begin{abstract}
The purpose of this study was to examine the physical match profiles of professional soccer players using 3 and 5 min fixed and rolling averages as well as fixed 1 min averages, with considerations to training prescription. Twenty-nine, professional U23 soccer outfield players competed across 17 competitive matches during the 2017/18 season, equating to a total of 130 separate physical match profiles. Match activities were recorded using global positioning system (GPS) devices with integrated micro-electrical mechanical systems (MEMS), recording total distance (TD), high-speed running (HSR) and metabolic power (MP). For each individual match profile and variable, 1, 3 and 5 min peak, post-peak, and average values were calculated using fixed-time epochs (FIXED) and rolling averages (ROLL). Linear mixed models were employed to examine the differences in the dependent variables as a function of the method of measurement. Results revealed significantly higher peak values, for relative TD, relative HSR and relative MP when employing the ROLL sampling method, in comparison to the FIXED method, for both $3 \mathrm{~min}$ and 5 min epoch lengths. Analysis of epoch length revealed significantly higher peak values, across all positions, for relative TD, relative HSR and MP for 1 min epochs, in comparison to $3 \mathrm{~min}$ and $5 \mathrm{~min}$ epochs. The data offers a novel insight into the appropriate identification of physical demands during youth soccer match-play. Researchers and practitioners should consider the sampling method and epoch length when assessing the physical demands of competitive match-play, as well as when designing and prescribing sport-specific conditioning drills.
\end{abstract}




\section{Introduction}

The application and use of technology in soccer has grown exponentially in recent years (Malone et al., 2017), allowing practitioners and researchers to evaluate the physical demands of competitive match play. This enables the prescription of appropriate training programmes, consistent with the physical requirements of competitive match-play, which requires players to perform high-intensity linear and multi-directional activities, interspersed with inconsistent periods of recovery (Cunningham et al., 2018; Delaney et al., 2017; Gabbett, 2015). Moreover, research has demonstrated the increase in the physical demands and intensity of competitive adult soccer match-play, across several seasons (Barnes et al., 2014; Bush et al. 2015). Indeed, Barnes et al. (2014) reported increases of $\sim 30 \%$, for high intensity running distance, and $\sim 50 \%$ for high intensity actions, across a 7-season period. Within this, however, positional differences in match-demands still exist, with central defenders showing a smaller increase in highintensity activity in comparison to midfielders, wide defenders, and attackers (Bush et al., 2015; Delaney et al., 2017). Consequently, training ought to be appropriately aligned to the demands of competition, so that players are suitably prepared for competitive match-play and more specifically, the peak periods of soccer match play. Currently, however, there is limited information regarding the peak demands of competitive match-play of youth soccer players. Information regarding the physical match demands of youth soccer is key for optimal talent development pathways, thus enabling the provision of age-appropriate training programmes, rather than extrapolating data from adult players.

Initially, assessment of the physical demands during competitive soccer match-play was investigated using discrete fixed-time epochs (e.g., 0-5, 5-10, 10-15 min), of differing lengths (Mohr, Krustrup \& Bangsbo, 2005). Indeed, research has examined changes in the physical demands during soccer match-play using both 15-min and 5 min epochs (Bradley et 
al., 2009; Mohr, Krustrup \& Bangsbo, 2003), in an attempt to provide a better understanding of the most intense periods of competitive match play (Bradley et al., 2009; Mohr et al., 2005). Since Varley, Elias and Aughey (2012), others have argued that both researchers and practitioners should analyse the physical demands of competitive matches using a rolling average (e.g., 0-5, 1-6, 2-7 min), due to issues of reduced sampling resolution associated with using pre-defined fixed-time epochs (Cunningham et al., 2018; Delaney et al., 2017; Doncaster et al., 2016). Despite the increased trend for adopting rolling averages for the examination of peak demands in team sports (Whitehead et al., 2018), there is limited research that has directly assessed differences between discrete fixed-time epochs and rolling averages, when examining the peak demands of competitive soccer match-play. Research in rugby union (Cunningham et al., 2018) identified that movement demands were consistently underestimated within fixedtime epochs for relative distance $\left(\mathrm{m} \cdot \mathrm{min}^{-1} ; \sim 11 \%\right.$ ) and high-speed running (HSR; 20\%), when compared to rolling averages.

Moreover, research has examined the physical demands of competitive match-play across differing time epochs, ranging from 1 to 10 min (Cunningham et al., 2018; Delaney et al., 2017; Whitehead et al., 2018). Indeed, training intensities for shorter durations (e.g. 1 and 3 min) should not be simply extrapolated from data obtained from longer epochs (Delaney et al., 2017). Both Cunningham et al. (2018) and Delaney et al., (2017) found that as the length of an epoch increased, both the relative intensity of the running actions and variability of the team physical output (e.g. HSR) decreased. Despite these comparable trends, the rate of decline in relative total and relative high-speed running distances, from 1 to $5 \mathrm{~min}$, was far greater in professional rugby union players (Cunningham et al., 2018) when compared to professional soccer players (Delaney et al., 2017). Given research has yet to examine whether similar patterns exist during youth soccer match play, there is a lack of knowledge and understanding 
with regards to the peak physical demands during youth soccer match-play. An improved understanding of the influence of sampling processes (i.e. fixed vs. rolling) and epoch length (i.e. 1 to $5 \mathrm{~min}$ ) has on the physical demands of match-play, in youth soccer, is required for optimal training prescription as well as for developing appropriate talent identification and development programmes.

To optimally prepare players for the demands of competitive youth soccer match-play, it is essential the most intense periods of competition are appropriately identified, for the respective age-groups. Therefore, the purpose of this study was to examine the differences in the physical demands of youth soccer players in relation to a) sampling method (fixed and rolling) and b) epoch length (1, 3 and 5 min splits), across the entire team and for each positional group.

\section{Methods}

\section{Participants}

Outfield players who competed within the respective Elite Player Performance Plan (EPPP), Category 1 Football Academy, Under 23 team $(n=29)$ were included in the study. Due to the potential for pacing strategies relating to players that are substituted (Waldron \& Highton, 2014), only those who played for the full duration of each match (90 min) were included for further analysis. As a result, a total of 130 separate, $90 \mathrm{~min}$, physical match profiles were recorded during the 2017/18 competitive season (Central Defenders $[\mathrm{CB}]=41$, Fullbacks $[\mathrm{FB}]=11$, Wing-backs $[\mathrm{WB}]=15$, Central Midfielders $[\mathrm{CM}]=43$, Wide Midfielders $[\mathrm{WM}]=9$ and Attackers $[\mathrm{ATT}]=11)$, from 16 different matches. Throughout, players' match activities, including total distance (TD), high-speed running (HSR: $\geq 19.8 \mathrm{~km} \cdot \mathrm{h}^{-1}$ ) and MP (regarded as the metres covered above a threshold of $25.5 \mathrm{~W} \cdot \mathrm{Kg}^{-1}$ but below $19.8 \mathrm{~km} \cdot \mathrm{h}^{-1}$ ) were 
recorded using $10 \mathrm{~Hz}$ devices with incorporated GPS receivers and MEMS technology (Viper Pod, STATSport, Belfast, UK). Total distance provides a measure of the volume of movement undertaken, whereas measures of HSR and MP provide an indication of the intensity of competitive match-play, with research showing a positive relationship between coaches' ratings of match performance and HSR (Bauer, Young, Fahrner \& Harvey, 2017). Metabolic power takes into consideration players' metabolic demands for high-speed accelerations and decelerations (Coutts et al., 2015), which may be missed if a single measure of HSR is employed. Data arose from the routine monitoring procedures employed within the respective club, with permission provided by the club and ethical approval granted from the local University Ethics Committee.

\section{Design \& Procedures}

Due to EPPP requirements for player monitoring during both training and competitive matches, all players were habituated with the devices and had extensive experiences of undertaking sport-specific activities and competing while wearing GPS units, with integrated MEMS technology. All matches were preceded by a standardized 25 min warm-up, which commenced approximately 40 min before kick-off and consisted of straight-line running and dynamic stretching of progressive intensity, followed by moderate and high intensity running with change of direction, before finishing with possession-based ball-work, which was interspersed with short periods of high-intensity activity. The units were fitted in a purpose made vest, leaving the units placed between the scapulae of each player during competitive match play. All units were switched on approximately 45 min before kick-off, allowing for an appropriate satellite signal to be achieved (Malone et al., 2017). After each match, minute-byminute 90 match profiles were downloaded and analysed using STATSport Software (119 STATSport Software), with confirmation of $>6$ satellites (an appropriate signal) being 
connected in all instances (Malone et al., 2017), before the minute-by-minute 90 min match profiles for TD, HSR and MP were inputted into a custom designed Microsoft Excel spreadsheet.

Despite the known within-game (Sparks, Coetzee \& Gabbett, 2017) and between-game (Doncaster \& Unnithan, 2017) variance in the physical demands of soccer match-play, the GPS and MEMS technology (STATsports Viper) have been shown to be reliable and valid when measuring distance and peak speed in sport related activities (Beato, Devereux \& Stiff, 2018). Indeed, Beato et al. (2018) reported high levels of reliability, showing 'small' coefficients of variation $(\mathrm{CVs})$ of $1.6 \%(90 \% \mathrm{CI}=1.3-2.3), 0.8 \%(0.7-1.2), 0.4 \%(0.3-0.5)$ and $0.7 \%(0.5$ - 0.9) for running distance, sport-specific distance, $20 \mathrm{~m}$ sprint distance and $20 \mathrm{~m}$ peak speed, respectively. Moreover, the validity of these units was reported to be 'good' with absolute errors of $7.9 \pm 7.2 \mathrm{~m}, 3.5 \pm 1.5 \mathrm{~m}, 20.2 \pm 0.3 \mathrm{~m}$ and $0.40 \pm 0.45 \mathrm{~km} \cdot \mathrm{h}^{-1}$, for 400 -m running trial, 128.5-m sport-specific circuit, 20-m sprint and peak speed.

For each of the given variables 1,3 and 5 min peak and post-peak values were calculated using fixed-time epochs (FIXED) and rolling averages (ROLL). FIXED epochs were calculated using previously established time epochs (e.g. 3 min splits: 1-3, 4-6, 7-9, etc. and 5 min splits: $1-5,6-10,11-15$, etc.), whereas ROLL epochs were calculated using rolling averages from the minute-by-minute 90 min physical match profiles (e.g. 3 min splits: 1-3, 24, 3-5, etc. and 5 min splits: 1-5, 2-6, 3-7, etc.) for 3 and 5 min epoch lengths. Post-peak split for FIXED epochs were then taken as the subsequent fixed-time epoch to the identified 'peak'. For ROLL epochs, however, the post-peak split was taken from the first minute after the previously identified 'peak period', for the respective amount of time (e.g. 3 min splits: peak $=$ 6-8 $\mathrm{min}$, post-peak $=9-11 \mathrm{~min}$ or 5 min splits: peak $=22-26 \mathrm{~min}$, post-peak $=27-31 \mathrm{~min})$. In 
addition, the mean average for TD, HSR and MP, for each epoch length (1, 3 and $5 \mathrm{~min})$ were calculated. With the mean average being calculated as the sum total of all epochs, dived by the respective number of epochs. To aid practical application and to enable comparison to be made between splits of different lengths ( 1 min vs. 3 min vs. 5 min), information was recorded as a relative measure $\left(\mathrm{m} \cdot \mathrm{min}^{-1}\right)$ for each of the given variables.

\section{Statistical Analysis}

As the collected data consisted of repeated measurements from the same group of players across a range of matches, linear mixed models (LMM) were used to examine the differences in the dependent variables (e.g. TD, HSR and MP) as a function of the method of measurement. A LMM was utilised to overcome the assumption of independence, and also because of the flexibility that this method has in accounting for the altering sample sizes between groups with repeated measures (Field, 2013). Before running the LMM, basic variance components analysis was performed, on each dependent variable, to calculate the intraclass correlations (ICC) of the random factors of player and opposition to determine if any contributed significant variance. Given the large sample sizes, Wald Z statistics were utilised to test the null hypothesis that the population variance is zero, if rejected the proposed random factors were included in subsequent larger models. The covariance structure of the random factors was set to variance components in all models. Model fit was assessed using Akaike's information criterion (AIC). For each dependant variable, AIC revealed the model that best fit the data utilising the first order auto-regressive (AR-1) repeated covariance structure for the repeated measures. The fixed effects and their interactions in each model included sampling method (FIXED and ROLL), epoch length (1, 3 and 5 min) and positional groups. All models estimated parameters using the maximum likelihood method. Where appropriate, Bonferroni adjusted post hoc analyses and the respective 95\% confidence intervals (95\% CI) of the 
differences were reported. Unless otherwise stated, data is presented as means with $95 \% \mathrm{CI}$. All statistical procedures were carried out using IBM SPSS Statistics (Version 25, Chicago, IL, USA), with two-tailed significance being accepted at $P<0.05$.

\section{Results}

Table 1 provides the ICC's (\%) of the random factors accounted for in each of the LMM. Where player and opposition contributed significant variance to the dependent variables, they were included in the larger hierarchical models, however, when they did not contribute any significant variance to the dependent variables, they were excluded as a random factor in the larger models.

\section{*** Insert Table 1 Here ${ }^{* * *}$}

The LMM did not identify significant interactions for position, epoch length and sampling method for relative TD $(P=0.881)$, relative $\operatorname{HSR},(P=0.997)$ and relative MP $(P=0.973)$ for the peak physical demands. Similarly, the LMM did not identify significant interactions for position, epoch length and sampling method for relative TD, relative HSR and MP, when analysing post-peak data and match average data. There were, however, main effects for position, epoch length and sampling method for relative TD, relative HSR and relative MP.

\section{Sampling Method}

Statistical analysis revealed significantly higher peak values, for relative TD, relative HSR and relative MP when employing the ROLL sampling method, in comparison to the FIXED method, for both $3 \mathrm{~min}$ (Mean Difference [95\% CI], TD = $6.3\left[4.5-8.0 \mathrm{~m} \cdot \mathrm{min}^{-1}\right], P<0.0001$; $\left.\mathrm{HSR}=2.9\left[1.1-4.6 \mathrm{~m} \cdot \mathrm{min}^{-1}\right], P=0.001 ; \mathrm{MP}=1.9\left[1.1-2.7 \mathrm{~W} \cdot \mathrm{kg}^{-1}\right], P<0.0001\right)$ and $5 \mathrm{~min}$ $\left(\mathrm{TD}=5.6\left[3.8-7.4 \mathrm{~m} \cdot \mathrm{min}^{-1}\right], P<0.0001 ; \mathrm{HSR}=2.6\left[0.8-4.3 \mathrm{~m} \cdot \mathrm{min}^{-1}\right], P=0.004 ; \mathrm{MP}=\right.$ 
$\left.1.7\left[0.9-2.5 \mathrm{~W} \cdot \mathrm{kg}^{-1}\right], P<0.0001\right)$ epoch lengths (Fig. 1A, 1B \& 1C). Conversely, analysis of the post-peak data revealed significantly lower values for relative MP, when employing the ROLL method, in comparison to the FIXED method, for both 3 min $\left(-2.1\left[-3.7\right.\right.$ to $-0.7 \mathrm{~W} \cdot \mathrm{kg}^{-}$ $\left.\left.{ }^{1}\right], P=0.005\right)$ and $5 \min \left(-1.5\left[-2.9\right.\right.$ to $\left.\left.0.1 \mathrm{~W} \cdot \mathrm{kg}^{-1}\right], P=0.047\right)$ epoch lengths. Similarly, both relative TD and relative HSR provided lower values when employing the ROLL method, in comparison to the FIXED method, for $3 \mathrm{~min}\left(\mathrm{TD}=-4.4\left[-1.2\right.\right.$ to $\left.10.1 \mathrm{~m} \cdot \mathrm{min}^{-1}\right], P=0.124$; $\mathrm{HSR}=-1.4\left[-3.1\right.$ to $\left.\left.0.2 \mathrm{~m} \cdot \mathrm{min}^{-1}\right], P=0.084\right)$ and $5 \mathrm{~min}\left(\mathrm{TD}=-3.2\left[-8.9\right.\right.$ to $\left.2.6 \mathrm{~m} \cdot \mathrm{min}^{-1}\right], P=$ $0.283 ; \mathrm{HSR}=-0.4\left[-2.1\right.$ to $\left.\left.1.2 \mathrm{~m} \cdot \mathrm{min}^{-1}\right], P=0.598\right)$; however, these were non-significant (Fig. 1D, $1 \mathrm{E} \& 1 \mathrm{~F})$. Finally, match averages for relative TD, relative HSR and relative MP were comparable between sampling methods, for both $3 \mathrm{~min}\left(\mathrm{TD}=0.3\left[-0.3\right.\right.$ to $\left.0.8 \mathrm{~m} \cdot \mathrm{min}^{-1}\right], P=$ $0.345 ; \mathrm{HSR}=0.1\left[-0.1\right.$ to $\left.0.3 \mathrm{~m} \cdot \mathrm{min}^{-1}\right], P=0.169 ; \mathrm{MP}=-0.1\left[-0.2\right.$ to $\left.\left.0.2 \mathrm{~W} \cdot \mathrm{kg}^{-1}\right], P=0.865\right)$ and $5 \mathrm{~min}\left(\mathrm{TD}=0.5\left[-0.1\right.\right.$ to $\left.1.1 \mathrm{~m} \cdot \mathrm{min}^{-1}\right], P=0.058 ; \mathrm{HSR}=0.1\left[-0.1\right.$ to $\left.0.2 \mathrm{~m} \cdot \mathrm{min}^{-1}\right], P=$ $0.226 ; \mathrm{MP}=0.1\left[-0.1\right.$ to $\left.\left.0.2 \mathrm{~W} \cdot \mathrm{kg}^{-1}\right], P=0.434\right)$.

\section{*** Insert Figure 1A, 1B, 1C, 1D, 1E \& 1F Here ***}

\section{Epoch Length \& Positional Comparisons}

Analysis of epoch length revealed significantly higher peak values, across all positions, for relative TD, relative HSR and MP for 1 min epochs, in comparison to 3 min and 5 min epochs. Likewise, although to a lesser extent, 3 min epochs were shown to produce significantly higher peak values for relative TD, relative HSR and MP, in comparison to 5 min epochs (Table 2). No significant differences were found across 1, 3 and 5 min epoch lengths for post-peak and match average data, across all variables. 
Results of the positional analysis of peak values, during different epoch lengths, are displayed in Figure 2A (relative TD), 2B (relative HSR) and 2C (relative MP). For peak relative TD, both CM (192.4 [189.6 - 196.1 $\left.\left.\mathrm{m} \cdot \mathrm{min}^{-1}\right]\right)$ and $\mathrm{WB}\left(192.4\left[187.6-197.1 \mathrm{~m} \cdot \mathrm{min}^{-1}\right]\right)$ were shown to have the highest values during 1 min epochs, however, as the epoch length increased only $\mathrm{CM}$ were shown to have the highest values $\left(3 \min =161.5\left[1581-164.9 \mathrm{~m} \cdot \mathrm{min}^{-1}\right] ; 5 \mathrm{~min}=151.2\right.$ $\left.\left[147.9-154.4 \mathrm{~m} \cdot \mathrm{min}^{-1}\right]\right)$. In contrast, ATT were shown to have the lowest values for peak relative TD for $1 \min \left(162.1\left[155.8-168.4 \mathrm{~m} \cdot \mathrm{min}^{-1}\right]\right), 3 \min \left(133.1\left[126.6-139.7 \mathrm{~m} \cdot \mathrm{min}^{-1}\right]\right)$ and $5 \mathrm{~min}\left(124.8\left[118.6-131.2 \mathrm{~m} \cdot \mathrm{min}^{-1}\right]\right)$ epochs. With regards to peak relative HSR, both WM $\left(64.4\left[58.7-70.2 \mathrm{~m} \cdot \mathrm{min}^{-1}\right]\right)$ and $\mathrm{WB}\left(64.9\left[60.9-68.9 \mathrm{~m} \cdot \mathrm{min}^{-1}\right]\right)$ were shown to have the highest values during 1 min epochs, with ATT (48.6 [43.5 - 53.8 $\left.\left.\mathrm{m} \cdot \mathrm{min}^{-1}\right]\right)$ displaying the lowest peak relative HSR values during 1 min epochs. As the epoch length increased though, $\mathrm{CB}$ reported the lowest values for peak relative $\operatorname{HSR}\left(3 \mathrm{~min}=22.5\left[19.7-25.3 \mathrm{~m} \cdot \mathrm{min}^{-1}\right] ; 5 \mathrm{~min}\right.$ $\left.=16.0\left[13.3-18.7 \mathrm{~m} \cdot \mathrm{min}^{-1}\right]\right)$. Finally, $\mathrm{CM}$ reported the highest values for peak relative MP, during $1 \min \left(45.6\left[44.3-47.0 \mathrm{~W} \cdot \mathrm{kg}^{-1}\right]\right), 3 \min \left(31.8\left[30.4-33.2 \mathrm{~W} \cdot \mathrm{kg}^{-1}\right]\right)$ and $5 \min (28.0$ $\left.\left[26.6-29.3 \mathrm{~W} \cdot \mathrm{kg}^{-1}\right]\right)$ epochs.

\section{*** Insert Figure 2A, 2B \& 2C Here***}

Analysis of post-peak relative TD data found that CM and WB performed significantly more relative TD, during 1 min epochs, when compared to CB (Mean Difference [95\% CI]: 28.6 $\left[15.5-41.8 \mathrm{~m} \cdot \mathrm{min}^{-1}\right], P<0.0001 ; 21.2\left[4.0-38.5 \mathrm{~m} \cdot \mathrm{min}^{-1}\right], P<0.0001$, respectively) and ATT $\left(32.5\left[11.4-53.5 \mathrm{~m} \cdot \mathrm{min}^{-1}\right], P<0.0001 ; 25.0\left[1.1-48.9 \mathrm{~m} \cdot \mathrm{min}^{-1}\right], P=0.031\right.$, respectively). As epoch length increased only $\mathrm{CM}$ were shown to perform significantly more relative $\mathrm{TD}$ than $\mathrm{CB}\left(3 \min =22.1\left[8.5-40.8 \mathrm{~m} \cdot \mathrm{min}^{-1}\right], P<0.0001 ; 5 \min =21.4[8.1-34.7\right.$ $\left.\left.\mathrm{m} \cdot \mathrm{min}^{-1}\right], P<0.0001\right)$ and ATT $\left(3 \mathrm{~min}=28.9\left[7.6-50.3 \mathrm{~m} \cdot \mathrm{min}^{-1}\right], P=0.001 ; 5 \mathrm{~min}=27.1\right.$ 
$\left.\left[3.9-50.2 \mathrm{~m} \cdot \mathrm{min}^{-1}\right], P=0.009\right)$. Post-peak data revealed limited differences between positions, for relative HSR and MP. Only a significant difference between CB and WM was found for HSR, during 1 min epochs, with WM performing a greater amount of HSR than CB (7.1 [0.5 $\left.\left.-13.6 \mathrm{~m} \cdot \mathrm{min}^{-1}\right], P=0.023\right)$. Whereas, only a significant difference between $\mathrm{CB}$ and $\mathrm{CM}$ was found for MP, during 1 min epochs, with $\mathrm{CM}$ displaying higher levels of $\mathrm{v}$ than $\mathrm{CB}$ (4.1 [0.6 $\left.\left.7.7 \mathrm{~m} \cdot \mathrm{min}^{-1}\right], P=0.009\right)$. There were no differences between positions, in relative TD, relative HSR and MP post peak data, for 3 and 5 min epochs.

Analysis of match averages for relative TD during 1, 3 and 5 min epochs revealed that CB (1 $\min =100.3\left[97.4-103.6 \mathrm{~m} \cdot \mathrm{min}^{-1}\right] ; 3 \mathrm{~min}=100.5\left[97.5-103.3 \mathrm{~m} \cdot \mathrm{min}^{-1}\right] ; 5 \min =101.1[98.2$ $\left.-103.9 \mathrm{~m} \cdot \mathrm{min}^{-1}\right]$ and ATT $\left(1 \mathrm{~min}=91.5\left[85.6-97.5 \mathrm{~m} \cdot \mathrm{min}^{-1}\right] ; 3 \mathrm{~min}=91.9[85.9-97.7\right.$ $\left.\mathrm{m} \cdot \mathrm{min}^{-1}\right] ; 5 \mathrm{~min}=92.7\left[86.7-98.7 \mathrm{~m} \cdot \mathrm{min}^{-1}\right]$ performed significantly less relative TD than FB $\left(1 \mathrm{~min}=106.0\left[102.5-109.5 \mathrm{~m} \cdot \mathrm{min}^{-1}\right] ; 3 \mathrm{~min}=106.5\left[102.9-110.3 \mathrm{~m} \cdot \mathrm{min}^{-1}\right] ; 5 \mathrm{~min}=107.3\right.$ $\left[103.9-110.7 \mathrm{~m} \cdot \mathrm{min}^{-1}\right], \mathrm{WB}\left(1 \mathrm{~min}=108.5\left[105.6-111.4 \mathrm{~m} \cdot \mathrm{min}^{-1}\right] ; 3 \mathrm{~min}=109.1[106.0-\right.$ $\left.112.4 \mathrm{~m} \cdot \mathrm{min}^{-1}\right] ; 5 \min =109.9\left[106.9-112.9 \mathrm{~m} \cdot \mathrm{min}^{-1}\right], \mathrm{CM}(1 \min =117.7[114.8-120.6$ $\left.\mathrm{m} \cdot \mathrm{min}^{-1}\right] ; 3 \mathrm{~min}=117.7\left[114.8-120.7 \mathrm{~m} \cdot \mathrm{min}^{-1}\right] ; 5 \min =117.9\left[115.1-120.9 \mathrm{~m} \cdot \mathrm{min}^{-1}\right]$ and WM, with FB and WB also performing significantly less relative total distance than CM, across all epoch lengths. In addition, WM were shown to perform significantly more relative TD than FB but only during 1 min epochs. With regards to match averages for relative HSR, CB (1 min $=5.4\left[4.6-6.1 \mathrm{~m} \cdot \mathrm{min}^{-1}\right] ; 3 \mathrm{~min}=5.4\left[4.6-6.1 \mathrm{~m} \cdot \mathrm{min}^{-1}\right] ; 5 \mathrm{~min}=5.5\left[4.7-6.3 \mathrm{~m} \cdot \mathrm{min}^{-1}\right]$ were shown to perform significantly less relative HSR than FB $\left(1 \mathrm{~min}=8.7\left[7.7-9.6 \mathrm{~m} \cdot \mathrm{min}^{-1}\right] ; 3\right.$ $\min =8.7\left[7.8-9.8 \mathrm{~m} \cdot \mathrm{min}^{-1}\right] ; 5 \min =8.9\left[8.0-9.8 \mathrm{~m} \cdot \mathrm{min}^{-1}\right], \mathrm{WB}(1 \mathrm{~min}=8.7[7.9-9.6$ $\left.\mathrm{m} \cdot \min ^{-1}\right] ; 3 \min =9.1\left[8.2-9.9 \mathrm{~m} \cdot \mathrm{min}^{-1}\right] ; 5 \min =9.5\left[8.7-10.3 \mathrm{~m} \cdot \mathrm{min}^{-1}\right], \mathrm{CM}(1 \mathrm{~min}=7.7$ $\left[7.0-8.5 \mathrm{~m} \cdot \mathrm{min}^{-1}\right] ; 3 \mathrm{~min}=7.7\left[6.9-8.5 \mathrm{~m} \cdot \mathrm{min}^{-1}\right] ; 5 \mathrm{~min}=7.8\left[7.0-8.5 \mathrm{~m} \cdot \mathrm{min}^{-1}\right]$ and $\mathrm{WM}$ $\left(1 \min =9.0\left[7.8-10.2 \mathrm{~m} \cdot \mathrm{min}^{-1}\right] ; 3 \mathrm{~min}=9.0\left[7.8-10.2 \mathrm{~m} \cdot \mathrm{min}^{-1}\right] ; 5 \min =9.1[7.9-10.2\right.$ 
$\mathrm{m} \cdot \mathrm{min}^{-1}$ ], across 1,3 and 5 min epochs. Beyond this, WB were shown to perform significantly more relative HSR than CM and ATT but only within the 5 min epochs. Finally, analysis of match averages for relative MP found CB $\left(1 \mathrm{~min}=12.0\left[11.2-12.8 \mathrm{~W} \cdot \mathrm{kg}^{-1}\right] ; 3 \mathrm{~min}=12.0\right.$ $\left.\left[11.2-12.9 \mathrm{~W} \cdot \mathrm{kg}^{-1}\right] ; 5 \mathrm{~min}=12.1\left[11.2-12.9 \mathrm{~W} \cdot \mathrm{kg}^{-1}\right]\right), \mathrm{FB}\left(1 \mathrm{~min}=12.8\left[11.8-13.9 \mathrm{~W} \cdot \mathrm{kg}^{-}\right.\right.$ $\left.\left.{ }^{1}\right] ; 3 \min =12.7\left[11.6-13.7 \mathrm{~W} \cdot \mathrm{kg}^{-1}\right] ; 5 \mathrm{~min}=12.7\left[11.7-13.7 \mathrm{~W} \cdot \mathrm{kg}^{-1}\right]\right)$ and ATT $(1 \mathrm{~min}=$ $\left.10.8\left[9.1-12.6 \mathrm{~W} \cdot \mathrm{kg}^{-1}\right] ; 3 \mathrm{~min}=10.9\left[9.2-12.7 \mathrm{~W} \cdot \mathrm{kg}^{-1}\right] ; 5 \mathrm{~min}=11.2\left[9.5-12.9 \mathrm{~W} \cdot \mathrm{kg}^{-1}\right]\right)$ to produce significantly lower values in comparison to WB $\left(1 \mathrm{~min}=14.5\left[13.6-15.3 \mathrm{~W} \cdot \mathrm{kg}^{-}\right.\right.$ $\left.\left.{ }^{1}\right] ; 3 \min =14.9\left[13.7-15.5 \mathrm{~W} \cdot \mathrm{kg}^{-1}\right] ; 5 \min =14.6\left[13.8-15.5 \mathrm{~W} \cdot \mathrm{kg}^{-1}\right]\right), \mathrm{CM}(1 \min =16.0$ $\left.\left[15.1-16.8 \mathrm{~W} \cdot \mathrm{kg}^{-1}\right] ; 3 \mathrm{~min}=15.9\left[15.0-16.8 \mathrm{~W} \cdot \mathrm{kg}^{-1}\right] ; 5 \min =15.9\left[15.0-16.8 \mathrm{~W} \cdot \mathrm{kg}^{-1}\right]\right)$ and $\mathrm{WM}\left(1 \mathrm{~min}=15.1\left[13.8-16.5 \mathrm{~W} \cdot \mathrm{kg}^{-1}\right] ; 3 \mathrm{~min}=15.2\left[13.9-16.5 \mathrm{~W} \cdot \mathrm{kg}^{-1}\right] ; 5 \mathrm{~min}=15.4\right.$ $\left.\left[14.1-16.6 \mathrm{~W} \cdot \mathrm{kg}^{-1}\right]\right)$, during 1,3 and 5 min epochs. Beyond this, CM were shown to report higher levels of relative MP, in comparison to WB but only within 1 min epochs.

\section{Discussion}

This study analysed the physical match demands of professional academy footballers using fixed and rolling 3 and $5 \mathrm{~min}$ epochs as well as comparing relative measures $\left(\mathrm{m} \cdot \mathrm{min}^{-1}\right)$ across 1, 3 and 5 min epochs, for the entire team and for each positional group. Our findings demonstrate that the ROLL method of analysis reports consistently higher intensities for both peak 3 and 5 min peak epochs, when compared to the traditional fixed sampling method. Conversely, analysis of post-peak and average 3 and 5 min epochs were comparable between fixed and rolling sampling methods. Moreover, we reaffirm previous findings demonstrating reductions in relative intensity as the epoch length increases (Cunningham et al., 2018; Delaney et al., 2017; Whitehead et al., 2018), with 1 min epochs producing the highest relative intensities for TD, HSR and MP, across all positions. 
Analysis of the physical demands in team sports has traditionally used a fixed method of analysing data from competitive match-play (Bradley et al., 2009; Bradley \& Noakes, 2013; Mohr et al., 2005). However, using a rolling average method of analysis, we report that peak relative TD, HSR and MP, during 3 and 5 min epochs, were higher in comparison to the fixed method in professional academy football players. Indeed, the fixed method underestimated relative TD distances, HSR distances and MP by up to $\sim 4.3 \%, \sim 11.8 \%$ and $\sim 7.4 \%$, respectively. These findings support the growing recognition that the adoption of fixed-time epochs might result in misidentifying the true 'peak' physical demands during competitive match-play, offering an inaccurate identification of players' peak physical demands during competitive match-play (Cunningham et al. 2018; Doncaster et al., 2016; Varley et al., 2012; Whitehead et al., 2018). Despite reporting a smaller discrepancy between the FIXED and ROLL methods, our data support the work of Cunningham et al. (2018) who reported underestimations of up to $\sim 11 \%$ and up to $\sim 20 \%$ for relative TD and HSR distances, respectively, when using a fixed sampling method in comparison to a rolling sampling method. Differences between the two studies are likely explained by differences in the sampling processes (i.e. second-by-second rather than the minute-by-minute) and the comparison of sporting demands and match profiles between different sports (rugby union vs. soccer). The higher values reported in the current study, across all variables for 3 and $5 \mathrm{~min}$ epochs, in comparison to those reported by Cunningham et al. (2018) will likely result in smaller percentage differences between sampling methods. Nevertheless, our data reaffirm the work of Cunningham et al. (2018) that highlights the impact sampling methods have upon the analysis of locomotor profiles and the identification and interpretation of the physical demands of soccer match-play. Furthermore, current findings suggest that the ROLL sampling method may also have implications for accurately identifying the post-peak periods of match-play, which is likely to have implication for training design and prescription. 
Relative measures $\left(\mathrm{m} \cdot \mathrm{min}^{-1}\right)$ of the demands during match play decreased as the epoch length increased. For example, overall team values were higher for 1 min epochs compared with 3 min epochs, with peak relative TD, HSR and MP displaying reductions 19.9\%, 52.2\% and $35.4 \%$, respectively, as epoch length increased. Similarly, but to a lesser extent, overall team values were higher for 3 min epochs compared with 5 min epochs, with TD, HSR and MP displaying reductions $6.1 \%, 24.4 \%$ and $13.2 \%$, respectively, as epoch length increased. Conversely, 'post-peak' relative values within the current study were lowest during the 1 min epochs. These data again highlight the extent to which epoch length can impact the 'peak' relative values and confirm the notion of the transient fatigue that follows in the 'post-peak' period of activity (Bradley et al. 2009; Cunningham et al., 2018; Delaney et al., 2017; Mohr et al., 2005). In support of Delaney et al. (2017), we show that rather than the 5 min sampling window used previously to assess the peak periods in soccer (Bradley et al. 2009; Mohr et al., 2005), the assessment of 'peak' periods using shorter epochs (i.e. $1 \mathrm{~min}$ ) may be more pertinent for the identification of 'peak' demands. As such, these findings can be used to inform and prescribe player appropriate and accurate training loads. Indeed, inappropriate processes in the sampling and identification of 'peak' demands could result in a substantial underestimation of the required intensity, thus resulting in the inappropriate design and application of training drills and players which are unable to meet the true 'peak' demands of competitive match-play.

This study also reports the positional differences for TD, HSR and MP dependent on sampling epoch length providing peak, post-peak, and average values. These data advance existing investigations into positional differences, which can be used for individualising training of positional groups in professional soccer. Previous research identified central midfielders and wide midfielders as the most physically demanding positions, in terms of 
locomotor activity (Bush et al., 2015; Bradley et al., 2009; Di Salvo et al., 2007). While current results support these findings, demonstrating that central and wide midfielders are physically demanding positions, our findings go further and suggest that more consideration toward the composition of what makes each of these positions physically demanding is required (e.g. consideration of 'peak' and 'post-peak' data). For example, while WB (192.4 [187.7 - 197.1 $\left.\left.\mathrm{m} \cdot \mathrm{min}^{-1}\right]\right)$ and $\mathrm{CM}\left(192.8\left[189.6-196.1 \mathrm{~m} \cdot \mathrm{min}^{-1}\right]\right)$ had similar peak relative TD values, during 1 min epochs, the post-peak relative TD values were different $(\mathrm{WB}=113.7[104.1-123.4$ $\left.\mathrm{m} \cdot \mathrm{min}^{-1}\right]$ vs. $\left.\mathrm{CM}=121.1\left[114.9-127.3 \mathrm{~m} \cdot \mathrm{min}^{-1}\right]\right)$. In addition, peak $1 \mathrm{~min}$ relative HSR values were greater for WB $\left(64.9\left[60.9-68.9 \mathrm{~m} \cdot \mathrm{min}^{-1}\right]\right)$ compared to CM $\left(54.5\left[51.9-57.1 \mathrm{~m} \cdot \mathrm{min}^{-}\right.\right.$ $\left.{ }^{1}\right]$ ), with similar relative HSR values being shown for each position, for 1 min post-peak epochs $\left(\mathrm{WB}=\left[3.3-9.16 .2 \mathrm{~m} \cdot \mathrm{min}^{-1}\right]\right.$ vs. $\left.\mathrm{CM}=\left[3.4-7.05 .2 \mathrm{~m} \cdot \mathrm{min}^{-1}\right]\right)$. Our findings highlight that both the epoch length, post-peak values and the specific metric, requires careful consideration when monitoring, evaluating and prescribing position-specific conditioning drills. Rather than simply prescribing a training drill according to the average values (which are similar irrespective of epoch length), the current findings emphasise the need to appreciate both the peak demands and post-peak demands, for individual metrics, when creating and monitoring position-specific conditioning drills. Such an approach will have implications for specific movements demands, volume, intensity and work to rest ratio of position-specific conditioning drills.

The sample size (particularly for positional analyses) and match-to-match variance in the physical demands measured should be considered when reviewing and applying the results of the current paper. While a large number of individual match profiles $(n=130)$ were obtained in the current study, samples sizes across positions did vary. Notwithstanding, the current results provide an indication toward the positional variation, where there is a consistent trend 
that the ROLL method of analysis provides consistently higher values for both peak 3 and 5 min peak epochs, when compared to the traditional fixed method of analysis. The roll sampling method employed within the current study can be enhanced further, by obtaining the raw velocity profiles from each player and using these to develop second-by-second (as opposed to minute-by-minute) rolling epochs (Cunningham et al., 2017; Delaney et al., 2018). Moreover, while the metric of MP incorporates the accelerations and decelerations undertaken by a player, consideration toward the number of high accelerations and decelerations during 'peak' physical periods of soccer match-play should be sought, thus enhancing training design and prescription (Delaney et al., 2017). Finally, further appreciation toward the impact match-to-match variance can have on 'peak' demands is needed. Indeed, the quality of the opposing team (Gabbett, 2013), phase of the season (Gregson et al. 2010; Kempton, Sullivan, Bilsborough, Cordy \& Coutts, 2015), weather conditions, substitutions, context of the match (win/lose margin) and current form (Black \& Gabbett, 2014) are all likely influences of the physical demands of match-play and need to be considered when analysing and assessing soccer match-play. Previous research, however, has tended to focus on the variance of match averages (i.e. across $90 \mathrm{~min}$ ) when assessing the physical demands of soccer match-play, rather than 'peak' periods of activity. Future research should seek to examine whether the same match-to-match variances exist for 'peak' and 'post-peak' epochs.

Given the widespread use of GPS and MEMS derived data, for the analysis and monitoring of the physical demands during competitive match-play in professional soccer, there is a need to ensure that the data obtained from such methods is appropriately gathered and interpreted. When looking to develop training drills which replicate the physical deamnds of soccer match-play, consideration toward sampling method and epoch length are needed. Indeed, the rolling sampling method should be utilised when seeking to identify the 'peak' 
physical demands during soccer match-play, irrespective of epoch length. In addition, the use of shorter epoch lengths, as commonly used within practice, is required for the identification of 'peak' physical loads and values should not be extrapolated from longer epoch lengths for the prescription of shorter training drills. Finally, position-specific physical loads should be prescribed, in which appreciation for post-peak demands should also be acknowledged.

\section{Conclusion}

Identifying the most intense periods of match-play provides informative data for training prescription and periodization, thus highlighting the importance of using appropriate methods of analysis when assessing the physical demands of competitive match-play. It appears that both method (FIXED vs. ROLL) and epoch length (1, 3 or $5 \mathrm{~min})$ are key variables for influencing the identification of the most intense periods (i.e. peak demands) of competitive match-play. When identifying the 'peak' physical demands, irrespective of epoch length (3 or $5 \mathrm{~min}$ ), a rolling method approach offers increased sensitivity. If the goal is to identify the peak physical demands during competitive match-play, then values using 1 min epochs (peak and post-peak) are recommended. Finally, practitioners are advised to align the epoch length to the length of the specific training drill that is being monitored or prescribed. For example, relative intensities from a $5 \mathrm{~min}$ epoch should not be extrapolated and utilised to assess and/or prescribe training drills which are longer or shorter in duration. Indeed, use of relative intensities obtained from a 5 min epoch, for a 1 or 3 min training drill, will result in an underestimation of an individual's physical capabilities and therefore an inappropriate training stimulus.

\section{Disclosure of Interest}

The authors report no conflicts of interest to report. 


\section{References}

Barnes, C., Archer D, T., Hogg, B., Bush, M., \& Bradley, P. (2014). The evolution of physical and technical performance parameters in English Premier League. International Journal of Sports Medicine. 35: 1-6.

Bauer, A., Young, W., Fahrner, B., \& Harvey, J. (2017). GPS variables most related to match performance in an elite Australian football team. international Journal of Performance Analysis in Sports, 15: 187-202. DOI: 10.1080/24748668.2015.11868786.

Beato, M., Devereux, G., \& Stiff A. (2018). Validity and reliability of global positioning system units (STATSports Viper) for measuring distance and peak speed in sports. Journal of Strength \& Conditioning Research, 32(10): 2831-2837.

Black, G., \& Gabbett, T. (2014). Match intensity and pacing strategies in rugby league: an examination of whole-game and interchanged players, and winning and losing teams. Journal of Strength \& Conditioning Research, 28(6): 1507-1516. DOI: 10.1519/JSC.0b013e3182a4a225.

Bradley, P., \& Noakes, T. (2013). Match running performance fluctuations in elite soccer: Indicative of fatigue, pacing or situational influences? Journal of Sports Science. 31(15): 16271638. http://dx.doi.org/10.1080/02640414.2013.796062.

Bradley, P, S., Sheldon, W., Wooster, B., Olsen, P., Boanas, P., \& Krustrup, P. (2009). Highintensity running in English FA Premier League soccer matches. Journal of Sports Science. 27(2): 159-168. DOI: 10.1080/02640410802512775. 
Bush, M., Barnes, C., Archer, D., Hogg, B., \& Bradley, P. (2015). Evolution of match performance parameters for various playing positions in the English Premier League. Human Movement Science. 39: 1-11. http://dx.doi.org/10.1016/j.humov.2014.10.003.

Coutts, A, J., Kempton, T., Sullivan, C., Bilsborough, J., Cordy, J., \& Rampinini, E. (2015). Metabolic power and energetic costs of professional Australian Football match-play. Journal of Science \& Medicine in Sport. 18: 219-224. http://dx.doi.org/10.1016/j.jsams.2014.02.003.

Cunningham, D, J., Shearer, D, A., Carter, N., Drawer, S., Pollard, B., Bennett, M........Kilduff, P. (2018). Assessing worst case scenarios in movement demands derived from global positioning systems during international rugby union matches: Rolling averages versus $\begin{array}{llll}\text { fixed length } & \text { epochs. } & \text { PLOSone. } & \text { 13(4): }\end{array}$ https://doi.org/10.1371/journal.pone.0195197.

Delaney, J., Thornton, H., Rowell, A., Dascombe, B, J., Aughey, R., \& Duthie, G. (2017). Modelling the decrement in running intensity within professional soccer players. Science \& Medicine in Football. 2(2): 86-92. DOI:10.1080/24733938.2017.1383623.

Di Salvo V, Baron R, Tschan H., Calderon Montero, F, J., Bachi, N., \& Pigozzi, F. (2007). Performance characteristics according to playing position in elite soccer. International Journal of Sports Medicine. 28(3): 222-227. DOI: 10.1055/s-2006-924294.

Doncaster, G., Marwood, S., Iga, J., Unnithan, V. (2016). Influence of oxygen uptake kinetics on physical performance in youth soccer. European Journal of Applied Physiology. 116: 17811794. DOI:10.1007/s00421-016-3431-x. 
Doncaster, G., \& Unnithan, V. (2017). Between-game variation of physical soccer performance measures in highly trained youth soccer players. Journal of Strength \& Conditioning Research, Epub ahead of print. DOI: 10.1519/JSC.0000000000002132.

Field, A. (2013). Discovering statistics using IBM SPSS statistics (4th ed.). London: Sage.

Gabbett, T. (2013). Influence of the opposing team on the physical demands of elite rugby league match play. Journal of Strength \& Conditioning Research, 27(6): 1629-1635. DOI: 10.1519/JSC.0b013e318274f30e.

Gabbett, T. (2015). Use of relative speed zones increases the high-speed running performed in team sport match play. Journal of Strength \& Conditioning Research, 29(12): 3353-3359.

Gregson W, Drust B, Atkinson G, Salvo V. (2010). Match-to-match variability of high speed activities in premier league soccer. International Journal of Sports Medicine, 31(4): 237-242. DOI: $10.1055 / \mathrm{s}-0030-1247546$.

Kempton, T., Sullivan, C., Bilsborough, J., Cordy, J., \& Coutts, A. (2015). Match-to-match variation in physical activity and technical skill measures in professional Australian Football. Journal of Science \& Medicine in Sport, 18(1): 109-113. DOI: 10.1016/j.jsams.2013.12.006.

Malone, J., Lovell, R., Varley, M, C., \& Coutts, A. (2017). Unpacking the black box: Applications and considerations for using GPS devices in sport. International Journal of Sports Physiology \& Performance. 12: S218-S226. http://dx.doi.org/10.1123/ijspp.2016-0236. 
Mohr, M., Krustrup, P., \& Bangsbo, J. (2003). Match performance of high-standard soccer players with special reference to the development of fatigue. Journal of Sports Sciences, 21: 519-528. DOI: 10.1080/0264041031000071182.

Mohr, M., Krustrup, P., \& Bangsbo, J. (2005). Fatigue in soccer: A brief review. Journal of Sports Science. 23(6): 593-599. http://dx.doi.org/10.1080/02640410400021286.

Sparks, M., Coetzee, B., \& Gabbett, T. (2017). Variations in high-intensity running and fatigue during semi-professional soccer matches. International Journal of Performance Analysis in Sport, 16: 122-132. DOI: 10.1080/24748668.2016.11868875.

Varley, M, C., Elias, G, P., \& Aughey, R, J. (2012). Current match-analysis techniques' underestimation of intense periods of high-velocity running. International Journal of Sports Physiology \& Performance. 7: 183-185. PMID: 22634968.

Varley, M, C., Gabbett, T., \& Aughey, R, J. (2013). Activity profiles of professional soccer, rugby league and Australian football match play. Journal of Sports Science. 32: 1858-1866. DOI:10.1080/02640414.2013.823227.

Waldron, M., \& Highton, J. (2014). Fatigue and pacing in high-intensity intermittent team sport: An update. Sports Medicine. 44(12): 1645-1658. DOI: 10.1007/s40279-014-0230-6.

Whitehead, S., Till., K., Weaving, D., \& Jones, B. (2018). The use of microtechnology to quantify the peak match demands of the football codes: a systematic review. Sports Medicine, 48(11). DOI: 10.1007/s40279-018-0965-6. 
Table 1: The ICC's (\%) of each random factor considering all of the dependent variables.

\begin{tabular}{lcc} 
Dependent Variable & Player & Opposition \\
\hline Relative Total Distance $\left(\mathbf{m} \cdot \mathbf{m i n}^{-1}\right)$ & & \\
Peak & $22.0^{*}$ & 4.1 \\
Post-Peak & $20.2^{*}$ & $9.1^{*}$ \\
Average & $73.7^{*}$ & $23.2^{*}$ \\
Relative HSR Distance $\left(\mathbf{m} \cdot \mathbf{m i n}^{-1}\right)$ & & \\
Peak & 4.1 & $25.7^{*}$ \\
Post-Peak & 7.3 & 0.1 \\
Average & $70.3^{*}$ & $13.0^{*}$ \\
Relative Metabolic Power $\left(\mathbf{W} \cdot \mathbf{K g}^{-1}\right)$ & & \\
Peak & $13.2^{*}$ & 1.3 \\
Post-Peak & $13.4^{*}$ & 4.8 \\
Average & $70.3^{*}$ & $17.5^{*}$ \\
Note: $*$ represent significant determinant of variance within the linear mixed model $(P<0.05)$.
\end{tabular}


Table 2: Comparison of relative TD, relative HSR and relative MP during peak 1, 3 and 5 min epochs.

\begin{tabular}{|c|c|c|c|c|c|c|}
\hline \multirow[b]{2}{*}{ Dependent variable } & \multirow{2}{*}{$\begin{array}{l}\text { Epoch } \\
\text { comparisons }\end{array}$} & \multirow{2}{*}{$\begin{array}{l}\text { Mean } \\
\text { Difference }\end{array}$} & \multirow[b]{2}{*}{ Std Error. } & \multirow[b]{2}{*}{$P$ Value } & \multicolumn{2}{|c|}{$\begin{array}{l}\text { 95\% Confidence Interval o } \\
\text { Difference }\end{array}$} \\
\hline & & & & & Lower & Upper \\
\hline \multicolumn{7}{|l|}{ Relative TD $\left(\mathrm{m} \cdot \mathrm{min}^{-1}\right)$} \\
\hline & 1 vs. 3 min & 35.9 & 0.9 & 0.0001 & 33.7 & 38.1 \\
\hline & 1 vs. 5 min & 44.8 & 1.1 & 0.0001 & 42.1 & 47.4 \\
\hline & 3 vs. 5 min & 8.8 & 0.9 & 0.0001 & 6.9 & 10.9 \\
\hline \multicolumn{7}{|l|}{ Relative HSR $\left(\mathrm{m} \cdot \mathrm{min}^{-1}\right)$} \\
\hline & 1 vs. $3 \mathrm{~min}$ & 29.4 & 0.9 & 0.0001 & 27.3 & 31.6 \\
\hline & 1 vs. 5 min & 36.2 & 1.0 & 0.0001 & 33.7 & 38.7 \\
\hline & 3 vs. 5 min & 6.8 & 0.9 & 0.0001 & 4.7 & 8.8 \\
\hline \multicolumn{7}{|c|}{ Metabolic Power (W.kg-1) } \\
\hline & 1 vs. $3 \mathrm{~min}$ & 14.2 & 0.4 & 0.0001 & 13.2 & 15.2 \\
\hline & 1 vs. 5 min & 17.5 & 0.5 & 0.0001 & 16.3 & 18.7 \\
\hline & 3 vs. 5 min & 3.3 & 0.4 & 0.0001 & 2.4 & 4.3 \\
\hline
\end{tabular}


Figure 1: Difference between FIXED and ROLL sampling methods for identifying peak (1A, 1B \& 1C) and post-peak $(1 \mathrm{D}, 1 \mathrm{E} \& 1 \mathrm{~F})$ demands of soccer match-play, during 3 and 5 min epochs. * indicates significantly different to FIXED epoch $(P<0.05)$.

Figure 2: Positional differences between peak demands of relative total distance (Fig 2A), relative HSR and relative metabolic power (2C), during 1, 3 and 5 min epochs. 